\title{
Paramedic interventions increase the rate of return of spontaneous circulation in out of hospital cardiac arrests
}

\section{Department of Accident and Emergency Medicine, Derriford Hospital, Plymouth PL6 8DH C J Mann H Guly}

Correspondence to: Dr C J Mann.

Accepted for publication 20 February 1997

\begin{abstract}
Objective-To determine whether paramedic interventions increased the rate of return of spontaneous circulation in the victims of out of hospital cardiac arrest. Methods-A retrospective analysis of 276 out of hospital cardiac arrests was made. Data analysed included age, sex, presenting rhythm, ambulance response time, presence of a pulse at any point, interventions performed by the ambulance crews, and survival to discharge.

Results-146 patients were treated by paramedics and 130 by technicians. There was no difference in the rate of return of spontaneous circulation or survival to discharge in patients presenting in ventricular fibrillation (VF). In non-VF arrests there was no increase in survival to discharge, but $15 \%$ of patients in non-VF arrests achieved a return of spontaneous circulation when treated by paramedics compared to none treated by technicians. There were no other significant differences in any of the variables assessed. Conclusions-Out of hospital cardiac arrests presenting in VF are managed equally well by paramedics and technicians. However, in non-VF arrests there is a significantly increased rate of return of spontaneous circulation in those patients attended by paramedics.

(F Accid Emerg Med 1997;14:149-150)
\end{abstract}

Keywords: out of hospital cardiac arrest; treatment; paramedic; technician

The survival to discharge of out of hospital cardiac arrest victims varies widely between $1.4 \%{ }^{1}$ and $20 \%$. $^{2}$ Important factors relating to survival are: (1) time before start of basic life support (that is, bystander cardiopulmonary resuscitation); (2) ambulance response times; and (3) a tiered ambulance response system. ${ }^{3}$ The presenting rhythm is also of vital importance since there is a very much better prognosis for ventricular fibrillation (VF) arrests than for non-VF arrests. For VF arrests, time to first defibrillation has also been shown to be a key determinant of survival. ${ }^{4}$

It has been Department of Health policy that since the end of 1995 all emergency ambulances in England and Wales should be manned by at least one trained paramedic, ${ }^{5}$ thus moving from a one tier system with technicians as first responders to a one tier system with paramedics as first responders. The value of this is unproven, and it has been suggested that paramedics are no more effective in the management of out of hospital cardiac arrests than ambulance technicians. ${ }^{6}$

Ambulance technicians are trained in defibrillation and basic airway management techniques, whereas paramedics can also use endotracheal intubation and intravenous cannulation, and can give adrenaline and atropine.

In this study we compared the outcome of cardiac arrests attended by paramedics and those attended by ambulance technicians.

\section{Methods}

Paramedics were introduced in the study area in 1992 and the percentage of ambulances manned by paramedics rose from $20 \%$ to $90 \%$ during the study period.

The clinical records of all out of hospital cardiac arrests were studied retrospectively for the three years 1993 to 1995 . The outcome measures used were return of spontaneous circulation and survival to discharge from hospital.

If a cardiac arrest was attended by ambulance technicians and subsequently by paramedics, it was excluded from this study. Similarly, arrests occurring in ambulances were also excluded.

\section{Results}

Two hundred and seventy six patients were entered into the study: 146 patients received treatment from paramedics (102 men, 44 women), median age 71 years (33 to 89 ); 130 patients were treated by technicians ( 88 men, 42 women), median age 70 years (29 to 88 ), P $>0.5$. The median response time for the paramedic group was 9 minutes (4 to 26 ) and for the technician group 8 minutes ( 3 to 30 ), $P=$ 0.8 .

Table 1 shows the cardiac rhythms at the time of ambulance arrival. There was no significant difference in the age, sex, ambulance response time, or presenting rhythms in the two groups.

Of the patients treated by paramedics, 59 $(42 \%)$ survived to return of spontaneous circulation. In the ambulance technician treated group, 34 patients $(26 \%)$ survived to return of spontaneous circulation $\left(\chi^{2}=3.95\right.$ : $P$ $=0.05$ ).

In patients in whom the presenting rhythm was VF there was a return of spontaneous circulation in 40 patients treated by ambulance 
Table 1 Presenting rhythms

\begin{tabular}{llll}
\hline & Paramedics & Technicians & P value \\
\hline Asystolic arrests & 50 & 43 & $>0.5$ \\
EMD arrests & 33 & 30 & $>0.5$ \\
VF arrests & 63 & 57 & $>0.5$ \\
Total arrests & 146 & 130 & $>0.5$ \\
$\begin{array}{l}\text { Return of spontaneous } \\
\quad \text { circulation }\end{array}$ & 59 & 34 & 0.05 \\
Survival to discharge & 4 & 5 & $>0.5$ \\
\hline
\end{tabular}

$\mathrm{EMD}$, electromechanical dissociation; VF, ventricular fibrillation.

Table 2 Return of spontaneous circulation

\begin{tabular}{llll}
\hline & All arrests & $V F$ & Non-VF \\
\hline Paramedics & $59(42 \%)$ & $40(27 \%)$ & $19(15 \%)$ \\
Technicians & $34(26 \%)$ & $34(26 \%)$ & $0(0 \%)$ \\
\hline
\end{tabular}

technicians and in 34 patients treated by paramedics (NS). However, in non-VF arrests there was a return of spontaneous circulation in none of the patients treated by ambulance technicians but in 19 of those treated by paramedics $(P=0.01)$ (table 2$)$.

Overall, nine patients survived to discharge from hospital $(3.2 \%)$. All but one of the patients who survived to discharge were in ventricular fibrillation at the scene.

\section{Discussion}

The decision by the Department of Health to put at least one trained paramedic on the crew of each emergency ambulance was not the result of research showing any specific benefits. In North America the use of ambulance technicians using defibrillators rather than full paramedic skills has been advocated. ${ }^{7-9}$

The prognosis for non-VF arrests is very poor. $^{71011}$ To date there is no evidence that paramedics have altered this.

Guly et al in Edinburgh ${ }^{6}$ found that paramedic attendance did not improve outcome from prehospital cardiac arrest when compared to a defibrillation trained technician. Their study has, however, been criticised ${ }^{1213}$ because the participating paramedics were not allowed to give cardioactive drugs (that is, adrenaline and atropine) and therefore could not be said to have performed advanced cardiac life support, as defined by the United Kingdom Resuscitation Council. This was not the case in this study.

This study confirms the results of an earlier study $^{6}$ in showing that the outcome for VF arrests is not improved by paramedic attendance. However, in non-VF arrests there was a significant increase in the number of patients with a return of spontaneous circulation when treated by paramedics, although this did not lead to an increased rate of survival to discharge.

\section{CONCLUSION}

At present, advanced cardiac life support as performed by paramedics has no advantages over basic life support with early defibrillation in the treatment of victims of out of hospital cardiac arrest. However, further research should be done to determine whether the return of spontaneous circulation in a significant number of non-VF arrests attended by paramedics represents the first step for better long term survival in these patients or merely prolongs dying.

The primacy of early defibrillation for VF arrests is again emphasised, and better management of out of hospital cardiac arrest may demand more radical methods of providing early defibrillation.

1 Lombardi G, Gallagher J, Gennis P. Outcome of out-ofhospital cardiac arrest in New York City. The Prehospital Arrest Survival Evaluation "study. JAMA 1994;271:67883.

2 Valenzuela TD, Spaite DW, Meislin HW. Case and survival definitions in out of hospital cardiac arrest on survival rate definitions in out of hospital cardiac a
calculations. JAMA 1992;267:272-4.

3 Nichol G, Detsky AS, Stiell IG, O'Rourke K, Wells G, Laupacis A. Effectiveness of emergency medical services Laupacis A. Effectiveness of emergency medical services
for victims of out of hospital cardiac arrest: a meta-analysis. for victims of out of hospital cardiac

4 American Heart Association. Guidelines for cardiopulmonary resuscitation (CPR) and emergency cardiac care (ECC). JAMA 1992;268:2172.

5 Annex to $\mathrm{HSG}^{92} \mathrm{XX}$. Parenteral administration of prescription only medicines by extended trained ambulance paramedics. London: HMSO, 1993.

6 Guly UM, Mitchell RG, Cook R, Steedman DJ, Robertson $\mathrm{CE}$. Paramedics and technicians are equally successful at managing cardiac arrest outside hospital. BMJ 1995;310: 1091-3.

7 Cummins RO, Ornato JP, Thies WH, Pepe PE. Improving survival from sudden cardiac arrest: the "chain of survival survival from sudden cardiac arrest: the "ch

8 Smith JP, Bodai BI, Geehr EC, Allen S. Pre-hospital cardiopulmonary resuscitation: a reappraisal. Can J Prehosp Med pulmonary resu

9 Stults KR, Brown DD. Defibrillation managed by EMTD's? Incidence and outcome without paramedic back-up. Am J Emerg Med 1986;4:491-5.

10 Cobbe SM, Redmond MJ, Watson JM, Hollingworth J Carrington DJ. Heartstart Scotland- initial experience of a national service for out of hospital defibrillation. BMJ 1991;302:1517-20.

11 Rowley JM, Mounser P, Garner C, Hampton JR. Advanced training for ambulance crews; implications from 403 consecutive patients with cardiac arrest managed by crews with simple training. BMJ 1987;295:1387-9.

12 Mindell J, Ward K, Ide S. Paramedics were not used effectively. BMJ 1995;311:508.

13 Weston C, Donnelly P. Studying only admissions is a source of potential bias. BMJ 1995;311:509. 